\title{
Letter regarding "Endovascular treatment of acute intracranial vertebrobasilar artery occlusion: a multicenter retrospective observational study"
}

\author{
Hongchen Zhao ${ }^{1}$ \\ Received: 15 October 2019 / Accepted: 18 November 2019 / Published online: 5 December 2019 \\ (C) The Author(s) 2019
}

Dear Editor-in-Chief:

I read with interest Quan et al.'s recent article, "Endovascular Treatment of Acute Intracranial Vertebrobasilar Artery Occlusion: A Multicenter Retrospective Observational Study" [1], which presented a multicenter retrospective observational study of endovascular treatment of acute intracranial vertebrobasilar artery occlusion (VBAO) to elucidate the efficacy of individualized endovascular treatment strategy for selected patients with acute VBAO. However, the last paragraph of the results asserts, "Of the 159 patients, 74 received tirofiban", which differs from the results shown in Table 3: "tirofiban $(n=85)$ ". Additionally, the indication of tirofiban is stated as "in patients treated using primary or rescue balloon angioplasty". However, this number is confusing given that "MT + rescue angioplasty $(n=43)$ " and "Angioplasty (or with stenting) ( $n=27)$ " suggests that 70 patients received tirofiban. Finally, Table 3 would be more convincing if a comparison of baseline characteristics between groups treated with or without tirofiban were supplied.

I would very much appreciate it if Quan et al. may explain the discrepancies noted above. Thank you for your attention.
Funding information No funding was received for this study.

\section{Compliance with ethical standards}

Conflict of interest I declare that I have no conflict of interest.

Ethical approval NA

Informed consent NA

\section{Reference}

1. Quan T et al (2019) Endovascular treatment of acute intracranial vertebrobasilar artery occlusion: a multicenter retrospective observational study. Neuroradiology. https://doi.org/10.1007/s00234-01902282-1

Publisher's note Springer Nature remains neutral with regard to jurisdictional claims in published maps and institutional affiliations.

This article is a reply to https://doi.org/10.1007/s00234-019-02282-1

Hongchen Zhao

17111220024@fudan.edu.cn

1 Department of Neurology, Huashan Hospital, Fudan University, Shanghai, No. 12 Middle Wulumuqi Road, Shanghai 200040, China 